\title{
Technology for NbN HEB based multipixel matrix of $\mathrm{THz}$ range
}

\author{
Ivan Tretyakov ${ }^{1}$, N. Kaurova ${ }^{1}$, S. Raybchun ${ }^{1}$ and G.N. Goltsman ${ }^{1}$
}

${ }^{1}$ Department of Physics, Moscow State University of Education, 29 Malaya Pirogovskaya St, Moscow, 119435, Russia

\begin{abstract}
The influence of homogeneity disorder degree of the thin superconducting $\mathrm{NbN}$ film across of $\mathrm{Si}$ wafer on characteristics of the Hot Electron Bolometers (HEB) has been investigated. Our experiments have been carried out near the superconducting transition and far below it. The high homogeneity disorder degree of the $\mathrm{NbN}$ film has been achieved by preparing the Si substrate surface. The fabricated HEBs all have almost identical $R(T)$ characteristics with a dispersion of Tc and the normal resistance $R_{300}$ of not more than $0.15 \mathrm{~K}$ and $2 \Omega$, respectively. The quality of the devises allows us to demonstrate clearly the influence of non-equilibrium processes in the S'SS' system on the device performance. Our fabrication technology also allows creating multiplex heterodyne and direct detector matrices based the HEB devices.
\end{abstract}

\section{[1] INTRODUCTION}

The disordered thin superconducting $\mathrm{NbN}$ film has found an application as a sensitive element in bolometric detectors of terahertz and infrared ranges, such as HEB devices [1], singlephoton SSPD detectors [2]. Modern instruments of observational astronomy SOFIA [3] and GUSTO [4] require the use of matrix detectors for the terahertz range. Each pixel of the matrix - an HEB - is now considered as a separate detector, requiring individual adjustment of the bias voltage and local oscillator (LO) power. With the matrix size of several dozen pixels, this approach is impractical from the point of view of Allan's time. A solution to the problem can become unified and not requiring individual adjustment HEB pixels. Modern HEBs as heterodyne detectors have practically reached their sensitivity limit [5,6]. However, the task of fabricating a relatively large number of similar HEBs remains unresolved. In this paper, we present the capabilities of our technology of $\mathrm{NbN} \mathrm{HEB}$ fabricating on $\mathrm{Si}$ substrates that allows us to obtain detectors with very close R (T) characteristics for geometrically identical detectors within one batch. The key aspects of our technology are the surface preparing process of the high-resistance Si substrate, as well as the process of cleaning the $\mathrm{NbN}$ contact areas before the $\mathrm{Au}$ deposition.

\section{[2] Results}

For our experiments the $\mathrm{NbN}$ film is deposited by the AJA ORION 8 unit for $69 \mathrm{sec}$. to achieve a thickness of $5 \mathrm{~nm}$. Fig. 1 shows a schematic of the HEB. The detector consists of a superconducting $\mathrm{NbN}$ film embedded into $\mathrm{Ti} / \mathrm{Au}$ terminals of a planar antenna deposited onto a $\mathrm{Si}$ substrate. The detector inner part consists of an $\mathrm{NbN}$ film between the antenna inner terminals, the so called $\mathrm{NbN}$ bridge with a given width $\mathrm{W}$ and length $\mathrm{L}$, and a multilayer structure of the $\mathrm{NbN} / \mathrm{Ti} / \mathrm{Au}$ antenna -outer terminals. The multilayer $\mathrm{NbN} / \mathrm{Ti} / \mathrm{Au}$ has a critical temperature $\mathrm{Tc} 2$ lower than the Tc1 of the $\mathrm{NbN}$ bridge.
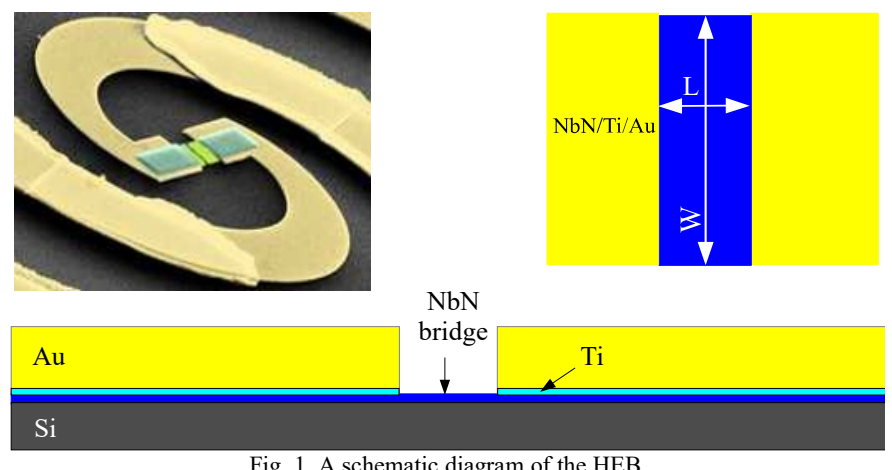

Fig. 1. A schematic diagram of the HEB.

The cleaning process of the Si substrate surface allows us to fabricate devices with a Tc1 deviation not more than $0.1 \mathrm{~K}$, for example, the in-situ technology presented in [7] gives a Tc1 dispersion of about $0,7-0,8 \mathrm{~K}$. Fig. 2 presents a family of $\mathrm{R}$ (T) characteristics of $\mathrm{NbN}$ HEBs. The similar shape of the $\mathrm{R}$ (T) curves demonstrates HEBs fabricated within in-situ $\mathrm{NbN} / \mathrm{Au}$ technology for HEB contacts [6]. The similarity of the Tc1 for the fabricated $\mathrm{NbN}$ HEBs across Si wafer gives the possibility to operate them at one common physical temperature as a single direct detector, or as a heterodyne detector with the same local oscillator power.

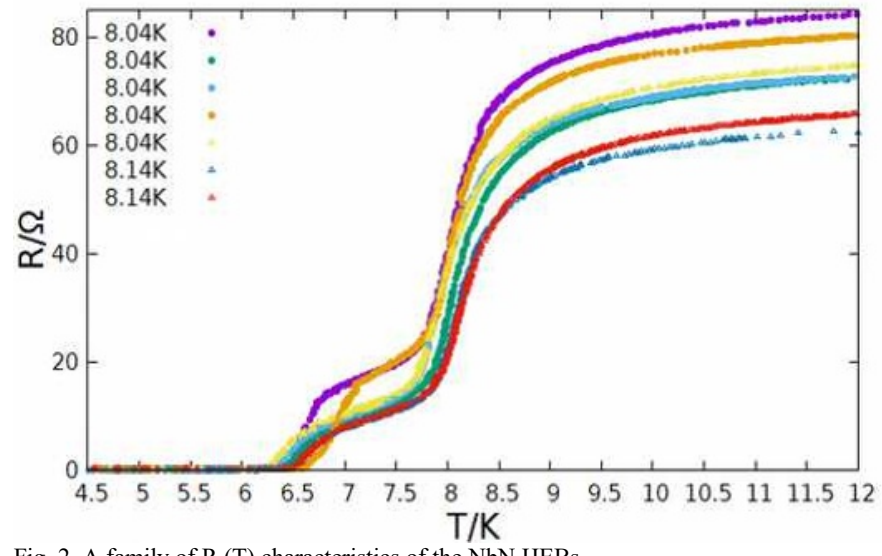

Fig. 2. A family of R (T) characteristics of the NbN HEBs.

The $\mathrm{NbN}$ film under $\mathrm{Ti} / \mathrm{Au}$ antenna ports was cleaned in $\mathrm{Ar}$ and $\mathrm{O} 2$ plasmas. The idea of cleaning was first proposed and applied for HEBs in [5]. This process made it possible to minimize the contact resistance between $\mathrm{NbN}$ and $\mathrm{Ti} / \mathrm{Au}$, and also to reduce the dispersion of the normal resistances to not more than $2 \Omega$ for detectors with the same $\mathrm{L} / \mathrm{W}$ ratio. The quality of the electrical contact between $\mathrm{NbN}$ and $\mathrm{Ti} / \mathrm{Au}$ explains the appearance of second transition on $\mathrm{R}(\mathrm{T})$ due to proximity effect. The HEBs resistance at $\mathrm{Tc} 1<\mathrm{T}<\mathrm{Tc} 2$ is built into the $\mathrm{NbN}$ superconducting bridge and is caused by the conversion of the normal electron current to the current of Cooper pairs over a length of oder $\xi$ [7]. Moreover, the charge conversion process also could influence the coordinate 
dependence of the energy gap near Tc1 [8]. It leads to a smearing of the superconducting transition and an increase of $\Delta \mathrm{Tc} 1$.

Fig. 3 shows a family of the IV curves of detector $1498 \_2$ \# 9 taken at the bath temperature ranging from $5 \mathrm{~K}$ to $\mathrm{T}>\mathrm{Tc} 1$. Fig. 3 we interprete as the evolution of the HEB IV curves upon transition from the S'SS' state [8], when the $\mathrm{NbN}$ bridge and the multilayer $\mathrm{NbN} / \mathrm{Ti} / \mathrm{Au}$ are in the superconducting state to the NSN [8] state where the $\mathrm{NbN}$ bridge is superconducting but multilayer $\mathrm{NbN} / \mathrm{Ti} / \mathrm{Au}$ is in the normal state.

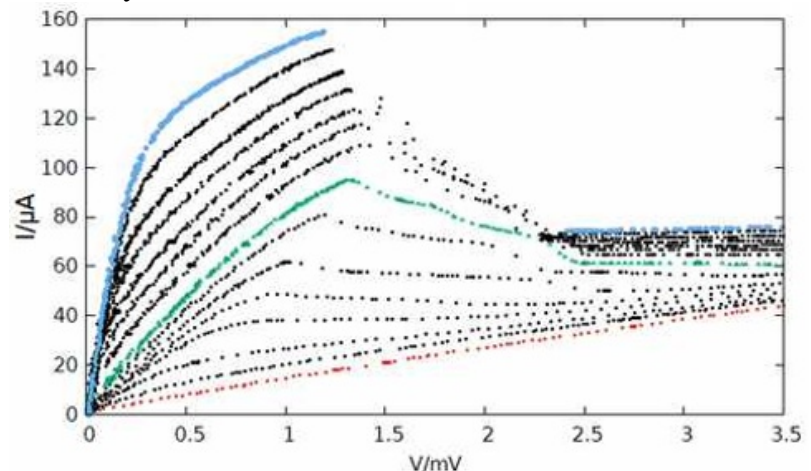

Fig. 3. A family of IV curves of detector $1498 \_2 \# 9$ in the temperature range from $5 \mathrm{~K}$ to $\mathrm{T}>\mathrm{Tc} 1$.

An HEB as a direct detector of $\mathrm{THz}$ radiation operates at the edge of the superconducting transition, being in the NSN state. In the mixer mode, an HEB operates at $\mathrm{T}<<\mathrm{Tc} 1$, being in the S'SS' state. The deviation in the measured minimal NEP of our HEB detectors is less than $15 \%$ at level of $5^{*} 10^{-13} \mathrm{~W} / \sqrt{\mathrm{Hz}}$. The dispersion of the HEBs optimal bias current and voltage is 3-5 $\mu \mathrm{A}$ and $0.1 \mathrm{mV}$ respectively. Such a small dispersion also indicates the identity of the HEBs. The measured values of the NEP are worse than the calculated value $1-2 * 10^{-14} \mathrm{~W} / \sqrt{ } \mathrm{Hz}$, which can be attributed to the influence of the processes at the metal/superconductor interface, which reduce the $\mathrm{dR} / \mathrm{dT}$ of the detector and give an additional contribution to the detector noise.

Fig. 4 presents the results of our study of HEB detectors as mixers in the S'SS' state. These mixers were fabricated using in-situ technology [6]. However, the surface of the Si substrate was not prepared in a special way before $\mathrm{NbN}$ deposition. The L/W bridge ratio was kept constant at 0.1-0.12 for all devices. Fig. 4 presents the dependence of the HEB noise temperature $\mathrm{Tn}$ measured at $2.5 \mathrm{THz}$ on the width of the $\mathrm{NbN}$ bridge. As can be seen from Fig. 4, there is a certain optimum volume of the $\mathrm{NbN}$ bridge with a minimum dispersion and a minimum $\mathrm{Tn}$ of $650 \mathrm{~K}$. The dispersion of $\mathrm{Tn}$ for devices with the same $\mathrm{L}$ and $\mathrm{W}$ fabricated from the same $\mathrm{NbN}$ film clearly demonstrates the influence of homogeneity disorder degree of the thin superconducting $\mathrm{NbN}$ film across of $\mathrm{Si}$ wafer on the characteristics of final devices. The optimal $\mathrm{W}$ indicates some optimal ratio of the $\mathrm{NbN}$ bridge heat capacity $\mathrm{C}$, the resistance coordinate dependences $\rho(\mathrm{L})$ and the distribution of the order parameter $\Delta(\mathrm{L})$ presented in Fig. 20a of [9]. For large W the optimal value the volume of the $\mathrm{NbN}$ bridge increases also as its $\mathrm{C}$, and accordingly the voltage responsivity $\mathrm{Sv}$ falls. The decrease of $\mathrm{W}$ of the $\mathrm{NbN}$ bridge leads to a proportional decrease of the $\rho(\mathrm{L})$ and the "hump" $\Delta(\mathrm{L})$ widths in Fig. 20a of [9].

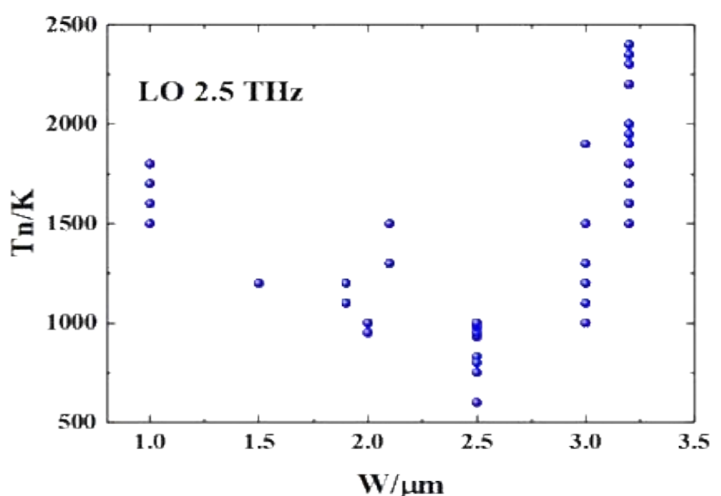

Fig. 4. The noise temperature Tn of an HEB bolometer as function of the width of the its superconducting $\mathrm{NbN}$ bridge.

Since, the absolute value of the "hump" $\Delta(\mathrm{L})$ width decreases, taking into account the diffusion length Le for a thin film of $\mathrm{NbN}$ in the resistive state $[6,10]$, some electrons absorbing the signal power, can escape into metal contacts not through the Andreev reflection but through tunneling, carrying energy with them - without a contribution to the deviation $\rho(L)$ and the the voltage responsivity of the entire system will fall.

\section{[3] CONCLUSION}

We demonstrated a fabrication technology of NbN HEB on $\mathrm{Si}$ substrates which allows us to obtain detectors with almost identical R ( $T$ ) characteristics and explain the optimal dimensions of the bridge for a low Tn. This technology allows the simple creating of the multiplex heterodyne and direct detector matrix based on HEB devices.

\section{ACKNOWLEDGEMENTS}

The research has been carried out with the support of the Russian Science Foundation (project No. 17-72-30036

\section{REFERENCES}

[1] E. M. Gershenzon, G. N. Goltsman, I. G. Gogidze, Y. P. Gusev, A. I. Elantiev, B. S. Karasik, and A. D. Semenov, "Millimeter and submillimeter range mixer based on electronic heating of superconducting films in the resistive state," Superconductivity, vol. 3, no. 10, pp. 1582-1597, 1990

[2] A. Semenov, G. N. Gol'tsman, R. Sobolewski, "Hot-Electron Effect in Superconductors and Its Applications for Radiation Sensors", LLE Review, V 87, pp 134-152, 2002.

[3] www.sofia.usra.edu

[4] www.sron.nl/missions-astrophysics/gusto

[5] J. J. A. Baselmans, M. Hajenius, J. R. Gao, T. M. Klapwijk, P. A. J. de Korte, B. Voronov, and G. Gol'tsman, "Doubling of sensitivity and bandwidth in phonon cooled hot electron bolometer mixers," Appl. Phys. Lett., vol. 84, pp. 1958-1960, 2004

[6] I. Tretyakov, S. Ryabchun, M. Finkel, A. Maslennikova, N. Kaurova, A. Lobastova, B. Voronov, and G. Goltsman, "Low noise and wide bandwidth of NbN hot-electron bolometer mixers," Appl. Phys. Lett., vol. 98, p. 033507, 2011.

[7] M. Shcherbatenko, I. Tretyakov, Y. Lobanov, S. N. Maslennikov, N. Kaurova, M. Finkel, B. Voronov, G. Goltsman, and T. M. Klapwijk, "Nonequilibrium interpretation of DC properties of $\mathrm{NbN}$ superconducting hot electron bolometers," Appl. Phys. Lett., vol. 109, p. 132602, 2016.

[8] N. Vercruyssen, T.G.A. Verhagen, M.G. Flokstra, J.P. Pekola, and T.M. Klapwijk, Phys.Rev. B85, 224503, 2012.

[9] T. M. Klapwijk and A. V. Semenov, "Engineering Physics of Superconducting Hot-Electron Bolometer Mixers," IEEE Transactions on Terahertz Science and Technology, vol/ 7, issue 6, pp. 627 - 648, 2017.

[10] A.D. Semenov, G.N. Goltsman, and A.A. Korneev, Physica C 351, 349, 2001 . 